\title{
Chemical differences and similarities among the kinematically selected thick disk, inner halo and outer halo stars
}

\author{
Miho N. Ishigaki ${ }^{1}$, Wako Aoki ${ }^{2}$ \& Masashi Chiba ${ }^{3}$ \\ ${ }^{1}$ Kavli Institute for the Physics and Mathematics of the Universe (WPI), University of Tokyo \\ 5-1-5 Kashiwanoha, 277-8583, Kashiwa, Japan \\ email: miho.ishigaki@ipmu.jp \\ ${ }^{2}$ National Astronomical Observatory of Japan, \\ 2-21-1 Osawa, 181-8588, Mitaka, Japan \\ email: aoki.wako@nao.ac.jp \\ ${ }^{3}$ Astronomical Institute, Tohoku University \\ 6-3 Aoba, Aramaki, 980-8578, Sendai, Japan \\ email:chiba@astr.tohoku.ac.jp
}

\begin{abstract}
Chemical diversity among metal-poor stars in the old stellar components in the Milky Way (MW), namely the thick disk and stellar halo, provides clues to understanding the early chemodynamical evolution of our Galaxy. We present our results on a homogeneous chemical abundance analysis for nearby metal-poor stars likely belonging to the MW thick disk, inner and outer stellar halos. Abundances of alpha, sodium, iron-peak and neutron-capture elements in the sample stars have been estimated using high-resolution $(R$ 50000) spectra obtained with the High Dispersion Spectrograph mounted on the Subaru Telescope. The derived abundances are used to examine differences and similarities in elemental abundance ratios among the kinematically defined thick disk, inner and outer halo subsamples in the metallicity range of $-3.3<[\mathrm{Fe} / \mathrm{H}]<$ -0.5 . We show that, in the metallicity range of $[\mathrm{Fe} / \mathrm{H}]<-2$, the three subsamples are similar in most of the elemental abundances. On the other hand, in the higher metallicities, particularly in $[\mathrm{Fe} / \mathrm{H}]>-1.5$, the thick disk and the inner/outer halo subsamples show systematically different abundance ratios for some elements including alpha, sodium, zinc and europium. A modest difference in the sodium and zinc abundances between the inner- and outer halo subsamples is also identified. The observed distinct abundances of some elements among the three subsamples implies that their constituent stars originally formed in progenitor systems that have experienced different star formation and chemical enrichment histories.
\end{abstract}

Keywords. stars: abundances, Galaxy: formation, Galaxy: halo

\section{Introduction}

The thick disk and stellar halo components of our Milky Way (MW) Galaxy largely consist of metal-poor stars and thus provide fossil records of the early chemodynamical evolution of the MW. Recent large surveys such as the Sloan Digital Sky Survey (SDSS) reveal that the MW stellar halo consists of at least two components (Carollo et al. 2010) as well as complex substructures, suggesting that the stellar halo formed through mergers of multiple progenitor systems.

Chemical diversity among the metal-poor stellar populations in the MW provide us with insights into accreted progenitor systems that are expected to have a variety of star formation and chemical enrichment histories. In particular, any correlation between chemical abundances and kinematics have been studiedas a clue to the origin of 


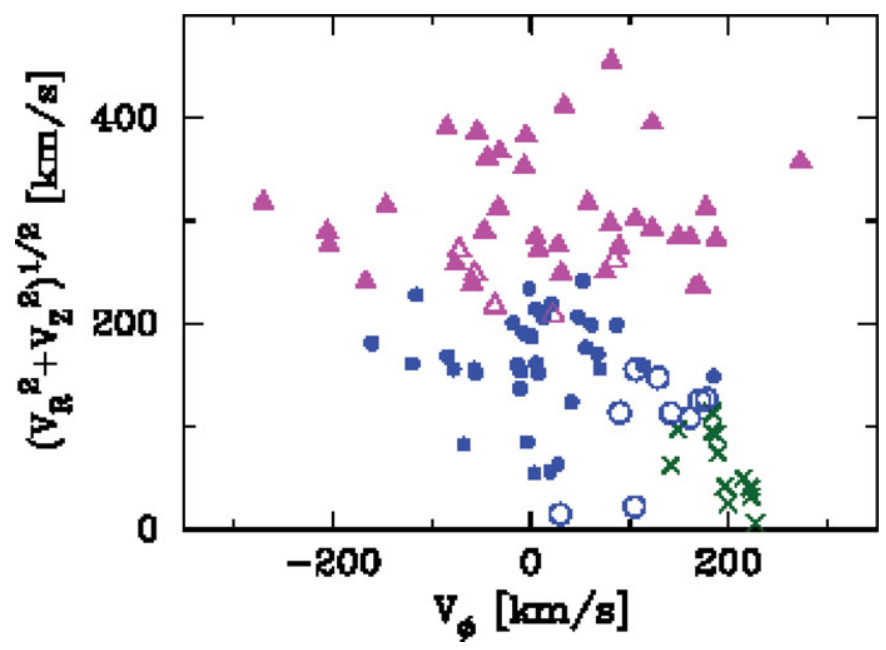

Figure 1. A Toomre diagram $\left(V_{\phi}\right.$ versus $\left.\left(V_{R}^{2}+V_{Z}^{2}\right)^{1 / 2}\right)$ for the sample stars. Crosses, filled circles and filled triangles indicate the sample stars with $P_{\mathrm{TD}}>0.9$ (the thick disk stars), $P_{\mathrm{IH}}>0.9$ (the inner halo stars) and $P_{\mathrm{OH}}>0.9$ (the outer halo stars), respectively. Open circles show the stars whose kinematics are intermediate between the thick disk and the inner halo $\left(P_{\mathrm{TD}}, P_{\mathrm{IH}} \leqslant 0.9\right.$ and $\left.P_{\mathrm{TD}}, P_{\mathrm{IH}} \geqslant P_{\mathrm{OH}}\right)$, while open triangles indicate stars whose kinematics are intermediate between the inner and the outer halo $\left(P_{\mathrm{IH}}, P_{\mathrm{OH}} \leqslant 0.9\right.$ and $\left.P_{\mathrm{IH}}, P_{\mathrm{OH}} \geqslant P_{\mathrm{TD}}\right)$.

metal-poor stars in the solar neighborhood (e.g. Stephens \& Boesgaard 2002,Fulbright 2002, Zhang et al. 2009). More recently, Nissen \& Schuster 2010 and Nissen \& Schuster 2011 reported that nearby halo stars include two populations with different chemical abundance properties, the low and high- $\alpha$ populations and chemical difference is likely related to their orbital properties.

In order to get deeper insights into the origin of the reported chemical difference among the nearby halo stars in the context of global formation mechanisms of the metal-poor components in the MW, we have carried out a homogeneous abundance analysis for a sample of nearby metal-poor stars that span wide ranges of orbital kinematics and metallicity.

\section{Observation and analysis}

\subsection{The sample}

The sample stars are selected from the catalogs of Carney et al. (1994), Ryan \& Norris (1991) and Beers et al. (2000), which include proper motions and radial velocity measurements as well as distance estimates. Their orbital parameters were calculated by the method of Chiba \& Beers (2000) All of the sample stars are nearby objects within $\sim 2$ $\mathrm{kpc}$ from the Sun.

The membership to the thick disk, inner halo and outer halo components are defined in this work using kinematics of the sample stars. For the membership assignment, we calculated probabilities at which each star belongs to the thick disk, inner halo and outer halo, adopting mean velocities and their scatter for each component from Carollo et al. (2010).

Velocity components in the Galactic cylindrical coordinate $\left(V_{R}, V_{\phi}\right.$ and $\left.V_{Z}\right)$ and the membership assignment are shown in Figure 1. 


\subsection{Observation}

The spectroscopic observations were carried out during 2008 to 2010 with the High Dispersion Spectrograph (HDS) mounted on the Subaru Telescope. Wavelengths of 4000$6800 \AA$ are covered with a spectral resolution of $R \sim 50000$. Data reductions were performed with standard IRAF routines.

\subsection{Abundance analysis}

Abundance analyses were performed with a one-dimensional LTE abundance analysis code (Aoki et al. (2009)), used with the Kurucz NEWODF model atmospheres. The abundances of sodium, $\alpha$-elements $(\mathrm{Mg}, \mathrm{Si}, \mathrm{Ca}$, and $\mathrm{Ti}$ ), iron-peak elements ( $\mathrm{Sc}, \mathrm{V}, \mathrm{Cr}$, $\mathrm{Mn}, \mathrm{Co}, \mathrm{Ni}, \mathrm{Cu}$ ), and neutron-capture elements ( $\mathrm{Sr}, \mathrm{Y}, \mathrm{Zr}, \mathrm{Ba}, \mathrm{La}, \mathrm{Sm}, \mathrm{Nd}, \mathrm{Eu}$ ) are estimated mainly based on the measured equivalent widths of absorption lines. For $\mathrm{Cu}$ and $\mathrm{Eu}$, a spectral synthesis technique is applied to estimate their abundances. More details of the abundance analysis procedure are presented in Ishigaki et al. (2012) and Ishigaki et al. (2013).

\section{Abundance results}

Figure 2 and 3 show abundance ratios $([\mathrm{X} / \mathrm{Fe}])$ plotted against $[\mathrm{Fe} / \mathrm{H}]$ for the thick disk(crosses), inner halo (filled circles) and outer halo (filled triangles) subsamples. More detailed results are presented in Ishigaki et al. (2012) and Ishigaki et al.(2013). In the following, we present the highlight results of our analysis, focusing on the similarity and difference among the three subsamples in individual abundance ratios.

$\alpha$ elements. In the range $[\mathrm{Fe} / \mathrm{H}]>-2.0$, the $[\mathrm{Mg} / \mathrm{Fe}]$ and $[\mathrm{Si} / \mathrm{Fe}]$ ratios in the inner and outer halo stars are lower on average with larger scatter than the thick disk subsample. At lower metallicities $([\mathrm{Fe} / \mathrm{H}]<-2)$, the three subsamples all show abundance ratios larger than the solar value.

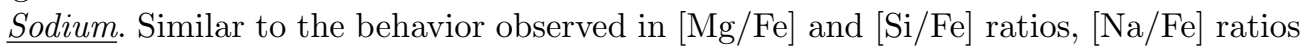
are lower in the inner and outer halo stars than in the thick disk stars in the range $[\mathrm{Fe} / \mathrm{H}]>-2.0$. There is a hint of an offset in the mean $[\mathrm{Na} / \mathrm{Fe}]$ ratios at $[\mathrm{Fe} / \mathrm{H}]>-2.0$ between the inner and outer halo stars in our sample.

Iron-peak elements. Differences between the thick disk and inner/outer halo stars are identified for $[\mathrm{Ni} / \mathrm{Fe}],[\mathrm{Cu} / \mathrm{Fe}]$ and $[\mathrm{Zn} / \mathrm{Fe}]$ ratios, again in the range $[\mathrm{Fe} / \mathrm{H}]>-2$. All of the three subsamples show increasing [Mn/Fe] abundance with increasing $[\mathrm{Fe} / \mathrm{H}]$. In this metallicity range, a modest difference in abundance ratios between the inner and outer halo stars can be seen in the $[\mathrm{Zn} / \mathrm{Fe}]$ ratios. In particular, stars with low$[\mathrm{Mg} / \mathrm{Fe}]$ abundances (gray circled symbols) show relatively low- $[\mathrm{Zn} / \mathrm{Fe}]$ abundance ratios consistent with the result reported by Nissen \& Schuster (2011).

Neutron-capture elements. In the metallicity range of $[\mathrm{Fe} / \mathrm{H}]<-2$, a hint of abundance difference between the thick disk and inner/outer subsample is identified for some of the light and heavy neutron-capture elements.

The Europium abundance was measured by fitting synthetic spectra to observed ones for $\mathrm{Eu}$ II lines (left panel of Figure 4). The difference in [Eu/Fe] ratios are identified but in an opposite sense of that seen in the $[\mathrm{Mg} / \mathrm{Fe}]$ ratios. As can be seen in the right panel of Figure 4, mean [Eu/Fe] ratios are higher for the inner/outer halo subsamples than in the thick disk subsample, while the $[\mathrm{Mg} / \mathrm{Fe}]$ ratios are lower for the inner/outer halo subsamples than in the thick disk subsample. This result is in contrast to the naive expectation that the $\mathrm{Eu}$ is produced in the astrophysical site similar to $\mathrm{Mg}$, which is largely produced in massive Type II SNe. 


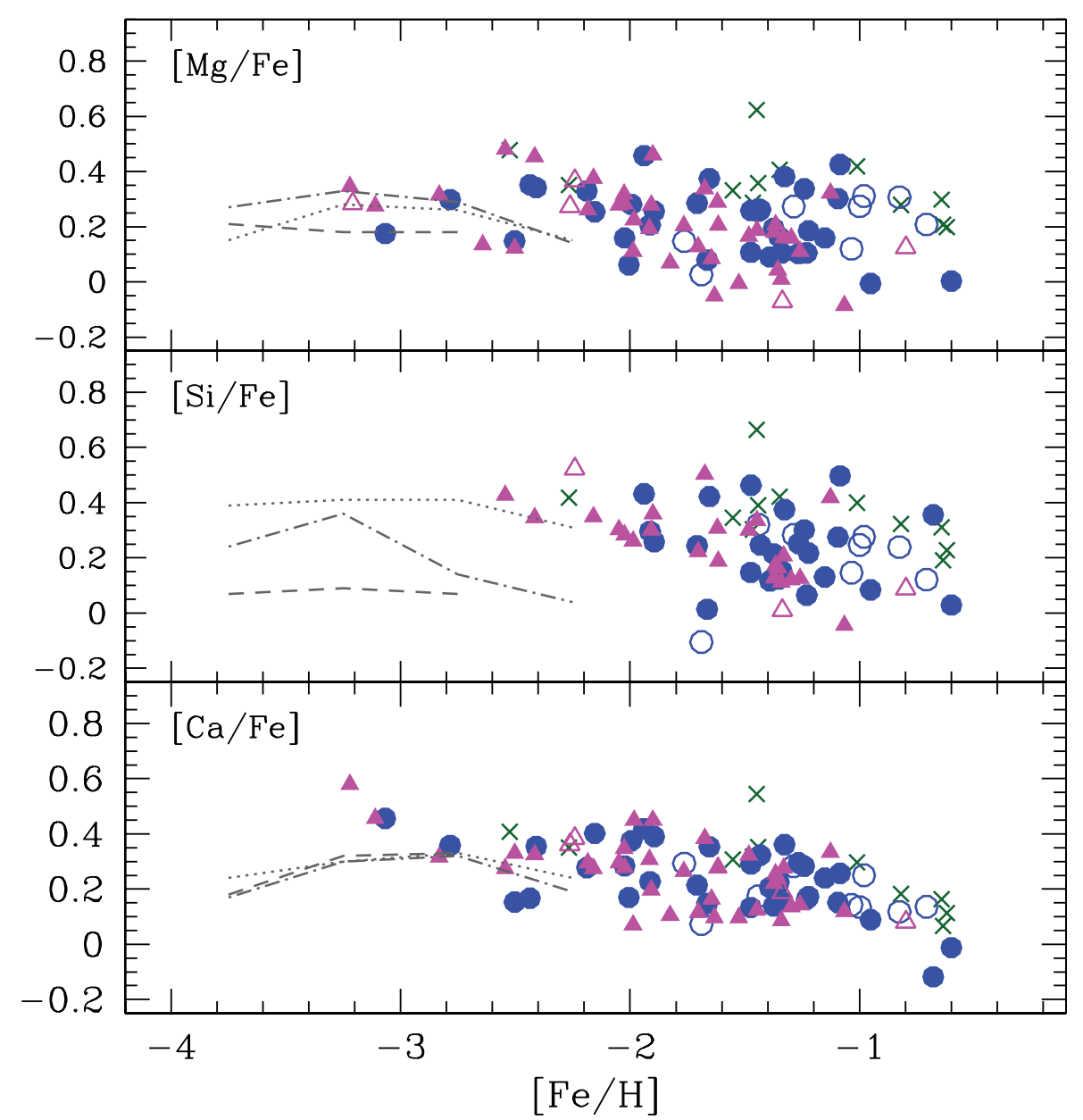

Figure 2. The abundance ratios of $\mathrm{Mg}, \mathrm{Si}$, and $\mathrm{Ca}$ plotted against $[\mathrm{Fe} / \mathrm{H}]$ for the thick disk (filled triangles), inner halo (filled circles) and outer halo (filled triangles) subsamples. The open circle and open triangles represent the thick disk/inner halo and inner halo/outer halo intermediate populations, respectively.

\section{Implications}

The most important result in this study is that at metallicities above $[\mathrm{Fe} / \mathrm{H}] \sim-1.5$ to -2.0 , the kinematically defined thick disk, inner halo and outer halo subsamples show different abundance ratios for some elements. In addition, scatter in the abundance ratios are larger in the inner and outer halo subsamples than in the thick disk subsample for many elements.

The difference in mean and scatter between the thick disk and the inner/outer halo subsamples suggest that these stars were originally formed in progenitor systems that had undergone different chemical enrichment histories. In particular, the lower- $[\mathrm{Mg} / \mathrm{Fe}]$, $[\mathrm{Si} / \mathrm{Fe}],[\mathrm{Na} / \mathrm{Fe}]$, and $[\mathrm{Zn} / \mathrm{Fe}]$ ratios in the inner/outer halo subsamples than in the thick disk subsample may suggest for a larger contribution of Fe from Type Ia SNe and/or an initial mass function biased toward lower-mass stars in the progenitors of the inner/outer halos.

A modest difference in the elemental abundance ratios such as $[\mathrm{Na} / \mathrm{Fe}]$ and $[\mathrm{Zn} / \mathrm{Fe}]$ 

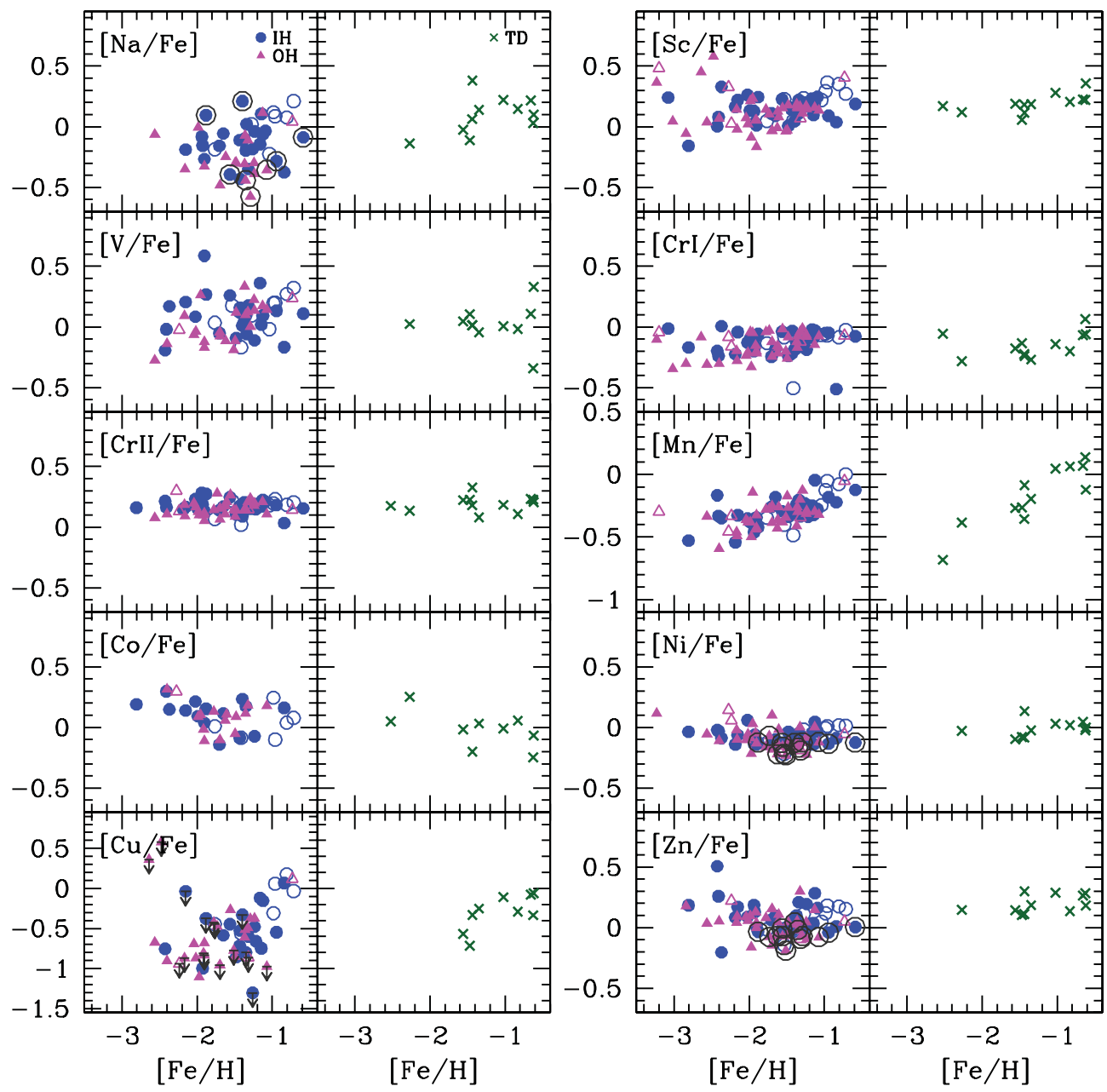

Figure 3. The abundance ratios of $\mathrm{Na}$ to $\mathrm{Zn}$ plotted against $[\mathrm{Fe} / \mathrm{H}]$. The symbols are the same as in Figure 3

between the inner and outer halo stars may further point to dominant progenitors of the metal-rich part of the outer halo is different from that of the inner halo.

At metallicities below $[\mathrm{Fe} / \mathrm{H}] \sim-2.0$, the three subsamples (mostly the inner and outer halo subsamples) show largely similar abundances. This implies that the star formation histories and chemical enrichment are similar among the progenitors of the inner and outer halos in this low metallicity range.

The observed abundance ratios are generally similar to those reported for dwarf satellite galaxies in the MW with similar metallicities, that are characterized by enhanced $[\alpha / \mathrm{Fe}]$ ratios and large scatter in abundances of neutron-capture elements. The similarity in abundances between the present inner/outer halo subsamples support the hypothesis that the large part of the stellar halo has been assembled from dwarf galaxies having similar star formation and chemical enrichment histories below $[\mathrm{Fe} / \mathrm{H}]<-2$ as suggested from previous analyses based on the SDSS photometric and spectroscopic data for a larger volume of the halo (e.g. Carollo et al. (2010), Schlaufman et al. (2012)). 

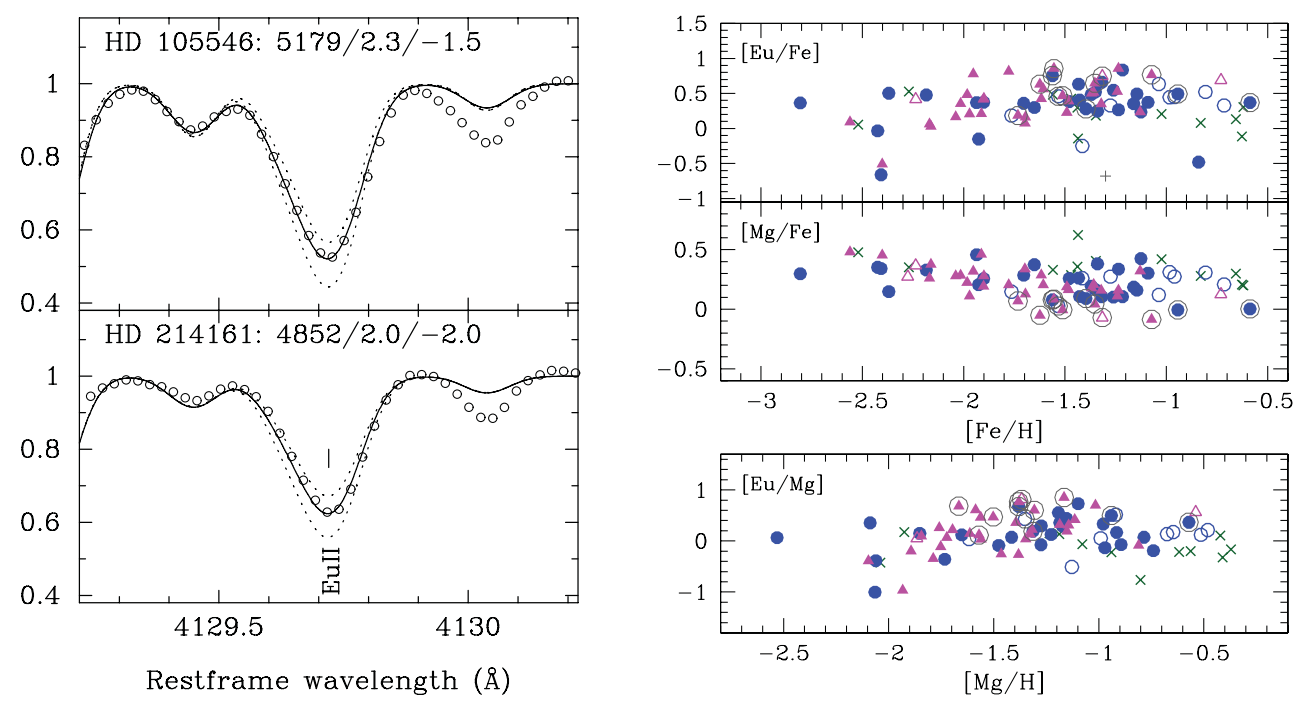

Figure 4. Left: Example synthetic and observed spectra for the Eu II line at $4129 \AA$ A. Right: ( Top and middle) The $[\mathrm{Eu} / \mathrm{Fe}]$ and $[\mathrm{Mg} / \mathrm{Fe}]$ ratios plotted against $[\mathrm{Fe} / \mathrm{H}]$ for the three subsamples. Symbols are the same as in Figure 2. (Bottom) $[\mathrm{Eu} / \mathrm{Mg}]$ ratios for the three subsamples.

\section{References}

Aoki, W., Barklem, P. S., Beers, T. C. et al. 2009, ApJ, 698, 1803

Beers, T. C., Chiba, M., Yoshii, Y. et al. 2000, AJ, 119, 2866

Carney, B. W., Latham, D. W., Laird, J. B. \& Aguilar, L. A. 1994, AJ, 107, 2240

Carollo, D., Beers, T. C., Chiba, M., et al. 2010, ApJ, 712, 692

Chiba, M. \& Beers, T. C. 2000, AJ, 119, 2843

Fulbright, J. P. 2002, AJ, 123, 404

Ishigaki, M. N., Chiba, M., \& Aoki, W. 2012, ApJ, 753, 64

Ishigaki, M. N., Aoki, W. \& Chiba, M. 2013, ApJ, 771, 67

Nissen, P. E. \& Schuster, W. J. 2010, A\&BA, 511, 10

Nissen, P. E. \& Schuster, W. J. 2011, $A \& A$, 530, 15

Ryan, S. G. \& Norris, J. E. 1991, AJ, 101, 1835

Schlaufman, K. C., Rockosi, C. M., Lee, Y. S., et al. 2012, ApJ, 749, 77

Stephens, A. \& Boesgaard, A. M. 2002, AJ, 123, 1647

Zhang, L., Ishigaki, M., Aoki, W., Zhao, G. \& Chiba, M. 2009, ApJ, 706, 1095

\section{Discussion}

Poul ERIK Nissen: Is there any possible nucleosynthesis explanation of the high [Eu/Mg] ratio in halo stars with $[\mathrm{Fe} / \mathrm{H}]>-2$ ?

Mino IshigAKI: The observed difference in the $[\mathrm{Eu} / \mathrm{Mg}]$ ratios between the thick disk and inner/outer halo subsamples suggest that Eu and $\mathrm{Mg}$ are largely produced in different astrophysical cites. One possibility is that r-process elements including Eu are preferentially synthesized in low-mass $\left(8-10 M_{\odot}\right)$ Type II SNe, while Mg is largely produced in more massive ones, as suggested from theoretical studies. 